\title{
Sign-Changing Solutions for Nonlinear Operator Equations
}

\author{
Yanbin Sang \\ Department of Mathematics, North University of China, Taiyuan, Shanxi 030051, China \\ Correspondence should be addressed to Yanbin Sang; sangyanbin@126.com
}

Received 20 December 2013; Accepted 29 January 2014; Published 6 March 2014

Academic Editor: Qingkai Kong

Copyright (C) 2014 Yanbin Sang. This is an open access article distributed under the Creative Commons Attribution License, which permits unrestricted use, distribution, and reproduction in any medium, provided the original work is properly cited.

The existence of six solutions for nonlinear operator equations is obtained by using the topological degree and fixed point index theory. These six solutions are all nonzero. Two of them are positive, the other two are negative, and the fifth and sixth ones are both sign-changing solutions. Furthermore, the theoretical results are applied to elliptic partial differential equations.

\section{Introduction}

In recent years, motivated by some ecological problems, much attention has been attached to the existence of signchanging solutions for nonlinear partial differential equations (see [1-4] and the references therein). We note that the proofs of main results in [1-4] depend upon critical point theory. However, some concrete nonlinear problems have no variational structures [5]. To overcome this difficulty, in [6], Zhang studied the existence of sign-changing solution for nonlinear operator equations by using the cone theory and combining uniformly positive condition.

$\mathrm{Xu}$ [7] studied multiple sign-changing solutions to the following $m$-point boundary value problems:

$$
\begin{aligned}
& y^{\prime \prime}(t)+f(y(t))=0, \quad 0 \leq t \leq 1, \\
& y(0)=0, \quad y(1)=\sum_{i=1}^{m-2} \alpha_{i} y\left(\eta_{i}\right),
\end{aligned}
$$

where $0<\alpha_{i}, i=1,2, \ldots, m-2,0<\eta_{1}<\eta_{2}<\cdots<\eta_{m-2}<1$.

We list some assumptions as follows.

$\left(A_{1}\right)$ Suppose that the sequence of positive solutions to the equation

$$
\sin \sqrt{x}=\sum_{i=1}^{m-2} \alpha_{i} \sin \eta_{i} \sqrt{x}
$$

is $\lambda_{1}<\lambda_{2}<\cdots<\lambda_{n}<\lambda_{n+1}<\cdots$;
$\left(\mathrm{A}_{2}\right) 0<\sum_{i=1}^{m-2} \alpha_{i}<1, f: \mathbb{R} \rightarrow \mathbb{R}$ is a continuous function, $f(0)=0$, and $x f(x)>0$ for all $x \in \mathbb{R} \backslash\{0\}$;

$\left(\mathrm{A}_{3}\right)$ let $\beta_{0}=\lim _{x \rightarrow 0}(f(x) / x)$ and $\beta_{\infty}=\lim _{|x| \rightarrow \infty}(f(x) /$ $x$ ). There exist positive integers $n_{0}$ and $n_{1}$ such that

$$
\begin{aligned}
& \lambda_{2 n_{0}}<\beta_{0}<\lambda_{2 n_{0}+1}, \\
& \lambda_{2 n_{1}}<\beta_{\infty}<\lambda_{2 n_{1}+1} ;
\end{aligned}
$$

$\left(\mathrm{A}_{4}\right)$ there exists $C_{0}>1$ such that

$$
|f(x)|<\frac{2\left(1-\sum_{i=1}^{m-2} \alpha_{i} \eta_{i}\right)}{5-\sum_{i=1}^{m-2} \alpha_{i} \eta_{i}} C_{0}
$$

for all $x \in \mathbb{R}$ with $|x| \leq C_{0}$.

Theorem 1 (see [7]). Suppose that conditions $\left(A_{1}\right)-\left(A_{4}\right)$ are satisfied. Then the problem (1) has at least two sign-changing solutions. Moreover, the problem (1) also has at least two positive solutions and two negative solutions.

Based on [7], many authors studied the sign-changing solutions of differential and difference equations. For example, Yang [8] considered the existence of multiple signchanging solutions for the problem (1). Compared with Theorem 1, Yang employed the following assumption which is different from $\left(\mathrm{A}_{4}\right)$.

$\left(\mathrm{A}_{4}^{\prime}\right)$ There exists $T>0$ such that

$$
|f(x)|<2(1-\alpha \eta)|x|, \quad|x| \leq T .
$$


Pang et al. [9] investigated multiple sign-changing solutions of fourth-order differential equation boundary value problems. Moreover, Wei and Pang [10] established the existence theorem of multiple sign-changing solutions for fourth-order boundary value problems. Y. Li and F. Li [11] studied two sign-changing solutions of a class of second-order integral boundary value problems by computing the eigenvalues and the algebraic multiplicities of the corresponding linear problems. He et al. [12] discussed the existence of signchanging solutions for a class of discrete boundary value problems, and a concrete example was also given. Very recently, Yang [13] investigated the following discrete fourth Neumann boundary value problems

$$
\begin{aligned}
& \Delta^{4} u(t-2)-\alpha \Delta^{2} u(t-1)+\beta(t)=f(t, u(t)), \\
& t \in[2, T]_{Z}, \\
& \Delta u(1)=\Delta u(T)=\Delta^{3} u(0)=\Delta^{3}(T-1)=0 .
\end{aligned}
$$

The author employed similar conditions with $\left(A_{1}\right)-\left(A_{4}\right)$ and obtained a similar result to Theorem 1 (see Theorem 5.1 in [13]).

The main purpose of this paper is to abstract more general conditions from $\left(A_{1}\right)-\left(A_{4}\right)$ of Theorem 1 , obtain the existence theorem of sign-changing solutions for general operator equations, and, then, apply the abstract result obtained in this paper to nonlinear elliptic partial differential equations.

\section{Preliminaries and Some Lemmas}

For the discussion of the following sections, we state here preliminary definitions and known results on cones, partial orderings, and topological degree theory, which can be found in $[14-18]$.

Let $E$ be a real Banach space. Given a cone $P \subset E$, we define a partial ordering $\leq$ with respect to $P$ by $x \leq y$ if and only if $y-x \in P$. A cone $P$ is said to be normal if there exists a constant $N>0$ such that $\theta \leq x \leq y$ implies $\|x\| \leq N\|y\|$; the smallest $N$ is called the normal constant of $P$. $P$ is called solid if it contains interior, that is, int $P \neq \emptyset$. If $x \leq y$ and $x \neq y$, we write $x<y$; if cone $P$ is solid and $y-x \in$ int $P$, we write $x \ll y . P$ is reproducing if $P-P=E$ and total if $\overline{P-P}=E$. Let $B: E \rightarrow E$ be a bounded linear operator. $B$ is said to be positive if $B(P) \subset P$. An operator $A$ is strongly increasing; that is, $x<y$ implies $A x \ll A y$. If $A$ is a linear operator, $A$ is strongly increasing which implies $A$ is strongly positive. A fixed point $u$ of operator $A$ is said to be a sign-changing fixed point if $u \notin P \cup(-P)$. If $x_{0} \in E \backslash\{\theta\}$ satisfies $\lambda A x_{0}=x_{0}$, where $\lambda$ is some real number, then $\lambda$ is called an eigenvalue of $A$ and $x_{0}$ is called an eigenfunction belonging to the eigenvalue $\lambda$.

Definition 2 (see [16]). Let $E_{1}, E_{2}$ be real Banach spaces and let $D \subset E_{1}$ contain the outside of a ball $\{x:\|x\| \leq r\}$, and $A: D \rightarrow E_{2}$. The operator $A$ is called asymptotically linear if there is a bounded linear operator $B: E_{1} \rightarrow E_{2}$ such that

$$
\lim _{\|x\| \rightarrow+\infty} \frac{\|A x-B x\|}{\|x\|}=0 .
$$

The operator $B$ involved in the definition of an asymptotically linear operator $A$ is uniquely determined. It is called the derivative of $A$ at infinity and is denoted by $A_{\infty}^{\prime}$.

Definition 3 (see $[16,18]$ ). Let $X$ be a retract of $E$, and let $U \subset$ $X$ be a relatively bounded open set of $X$. Suppose that $A$ : $\bar{U} \rightarrow X$ is completely continuous and has no fixed point on $\partial U$. Let the positive integer $i(A, U, X)$ be defined by

$$
i(A, U, X)=\operatorname{deg}\left(I-A r, T_{R} \cap r^{-1}(U), \theta\right),
$$

where $r: E \rightarrow X$ is an arbitrary retraction, and $R$ is a large enough positive number such that $\bar{U} \subset T_{R}=\{x \mid x \in$ $E,\|x\|<R\}$. Then $i(A, U, X)$ is called the fixed point index of $A$ on $U$ with respect to $X$.

Lemma 4 (see $[14,18]$ ). Let $P$ be a reproducing cone and let $B$ be a positive completely continuous linear operator, with $r(B)>$ 0 , where $r(B)$ denotes the spectral radius of $B$. Then $B$ has a positive eigenfunction in $P \backslash\{\theta\}$ corresponding to the eigenvalue $(r(B))^{-1}$.

Lemma 5 (see [18]). Let $P$ be a cone in $E$ and let $\Omega$ be a bounded open set of $E$, and $\theta \in \Omega$. Assume that $A: P \cap \bar{\Omega} \rightarrow P$ is a condensing operator. If $A x \nsupseteq x$ for every $x \in P \cap \partial \Omega$, then $i(A, P \cap \Omega, P)=1$.

Lemma 6 (see [19]). Let $P$ be a normal and total cone in $E$, and let $A: E \rightarrow E$ be a completely continuous increasing operator. Then the following assertions hold

(a) $A(\theta)=\theta, A$ is Fréchet differentiable at $\theta$. If $A_{\theta}^{\prime} x \neq x$, $\forall x \in P \backslash\{\theta\}$, and there exist $\lambda_{0}<1$ and $x_{0} \in P \backslash\{\theta\}$ such that $\lambda_{0} A_{\theta}^{\prime} x_{0}=x_{0}$, then there exists $\rho_{0}>0$ such that $i\left(A, B_{\rho} \cap P, P\right)=0$ for all $\rho \in\left(0, \rho_{0}\right]$, where $B_{\rho}=$ $\{x \in E \mid\|x\|<\rho\} ;$

(b) $A$ is an asymptotically linear operator. If $A_{\infty}^{\prime} x \neq x$, $\forall x \in P \backslash\{\theta\}$, and there exist $\tilde{\lambda}_{0}<1$ and $\tilde{x}_{0} \in P \backslash\{\theta\}$ such that $\tilde{\lambda}_{0} A_{\infty}^{\prime} \tilde{x}_{0}=\tilde{x}_{0}$, then there exists $\rho_{\infty}>0$ such that $i\left(A, B_{\rho} \cap P, P\right)=0$ for all $\rho \geq \rho_{\infty}$.

Lemma 7 (see [18]). Let $D$ be an open set of $E, A: D \rightarrow E$ be completely continuous, $x_{0} \in D$, and $A x_{0}=x_{0}$. Assume that $A$ is Fréchet differentiable at $x_{0}$ and 1 is not an eigenvalue of $A_{x_{0}}^{\prime}$, then $x_{0}$ is an isolated fixed point, and

$$
\text { ind }\left(I-A, x_{0}\right)=\operatorname{ind}\left(I-A_{x_{0}}^{\prime}, \theta\right)=(-1)^{\eta} \text {, }
$$

where $\eta$ is the sum of algebraic multiplicities of the real eigenvalues of $A_{x_{0}}^{\prime}$ in $(0,1)$.

Lemma 8 (see [18]). Suppose that $A: E \rightarrow E$ is a completely continuous and asymptotically linear operator. If 1 is not an eigenvalue of the linear operator $A_{\infty}^{\prime}$, then there exists $R_{0}>0$ such that

$$
\operatorname{deg}\left(I-A, B_{R}, \theta\right)=(-1)^{\gamma},
$$

for all $R \geq R_{0}$, where $\gamma$ is the sum of the algebraic multiplicities of the real eigenvalues of $A_{\infty}^{\prime}$ in $(0,1)$. 
Lemma 9 (see [9]). Let $X$ be a solid cone in $E, \Omega$ be a relatively bounded open subset of $X$, and $A: X \rightarrow X$ be a completely continuous operator. If any fixed point of $A$ in $\Omega$ is an interior point of $X$, then there exists an open subset $O$ of $E(O \subset \Omega)$ such that

$$
\operatorname{deg}(I-A, O, \theta)=i(A, \Omega, X) .
$$

Lemma 10 (see [18]). Let $\Omega$ be a bounded open set of $E$, and let $\theta \in \Omega$. Assume that $A: \bar{\Omega} \rightarrow E$ is a condensing operator. If

$$
A x \neq \mu x, \quad \forall x \in \partial \Omega, \mu \geq 1,
$$

then $\operatorname{deg}(I-A, \Omega, \theta)=1$.

\section{Multiple Sign-Changing Solutions for Nonlinear Operator Equations}

Theorem 11. Let $P$ be a normal solid cone in $E, A: E \rightarrow E$ be a completely continuous operator, $A(P \backslash\{\theta\}) \subset P^{\circ}, A(-P \backslash\{\theta\}) \subset$ $-P^{\circ}$, and $A \theta=\theta$. Suppose that

$\left(\mathrm{H}_{1}\right) A_{\infty}^{\prime}$ exists and is an increasing operator; $r\left(A_{\infty}^{\prime}\right)>1$; 1 is not an eigenvalue of $A_{\infty}^{\prime}$, and the sum of algebraic multiplicities for the real eigenvalues of $A_{\infty}^{\prime}$ in $(0,1)$ is an even number;

$\left(\mathrm{H}_{2}\right) A_{\theta}^{\prime}$ exists and is an increasing operator; $r\left(A_{\theta}^{\prime}\right)>1 ; 1$ is not an eigenvalue of $A_{\theta}^{\prime}$, and the sum of algebraic multiplicities for the real eigenvalues of $A_{\theta}^{\prime}$ in $(0,1)$ is an even number;

$\left(\mathrm{H}_{3}\right) \lim _{\|x\| \rightarrow 0} \sup (\|A x\| /\|x\|)<1$.

Then A has at least two sign-changing fixed points, two positive fixed points, and two negative fixed points.

Proof. From condition $\left(\mathrm{H}_{3}\right)$, we obtain that there exists $\tilde{r}>0$ such that $\|A x\|<\|x\|$ for all $0<\|x\|<\tilde{r}$. By Lemma 5 , there exists $r<\widetilde{r}$ such that

$$
\begin{gathered}
i\left(A, P \cap T_{r}, P\right)=1, \\
i\left(A,-P \cap T_{r},-P\right)=1,
\end{gathered}
$$

where $T_{r}=\{x \in E \mid\|x\|<r\}$.

Since $A(P \backslash\{\theta\}) \subset P^{\circ}, A(-P \backslash\{\theta\}) \subset-P^{\circ}$, and $A \theta=\theta$, which together with $\left(\mathrm{H}_{2}\right)$ imply that $A_{\theta}^{\prime}(P) \subset P$ and $r\left(A_{\theta}^{\prime}\right)>$ $1>0$. According to Lemma 4, we know that there exists $\bar{\varphi} \in P \backslash\{\theta\}$ such that $A_{\theta}^{\prime} \bar{\varphi}=r\left(A_{\theta}^{\prime}\right) \bar{\varphi}>\bar{\varphi}$. Since 1 is not an eigenvalue of $A_{\theta}^{\prime}, A_{\theta}^{\prime} x \neq x$ and $\forall x \in P \backslash\{\theta\}$. By Lemma 6 , there exists $\rho_{0} \in(0, r)$ such that

$$
i\left(A, P \cap T_{\rho}, P\right)=0
$$

for all $\rho \in\left(0, \rho_{0}\right]$. Similarly, we get that

$$
i\left(A,-P \cap T_{\rho},-P\right)=0 .
$$

In the same sense, we know that there exists $\rho_{\infty}>r$ such that

$$
\begin{gathered}
i\left(A, P \cap T_{\rho}, P\right)=0, \\
i\left(A,-P \cap T_{\rho},-P\right)=0,
\end{gathered}
$$

for all $\rho \geq \rho_{\infty}$. Further, combining Lemmas 7 and 8 with $\left(\mathrm{H}_{1}\right)$ and $\left(\mathrm{H}_{2}\right)$, we get that there exist $\rho_{1} \in\left(0, \rho_{0}\right)$ and $\rho_{2}>\rho_{\infty}$ such that

$$
\begin{aligned}
& \operatorname{deg}\left(I-A, T_{\rho_{1}}, \theta\right)=1, \\
& \operatorname{deg}\left(I-A, T_{\rho_{2}}, \theta\right)=1 .
\end{aligned}
$$

By (15)-(17), we have

$$
\begin{gathered}
i\left(A, P \cap T_{\rho_{1}}, P\right)=0, \\
i\left(A,-P \cap T_{\rho_{1}},-P\right)=0, \\
i\left(A, P \cap T_{\rho_{2}}, P\right)=0, \\
i\left(A,-P \cap T_{\rho_{2}},-P\right)=0 .
\end{gathered}
$$

It follows from (13), (20), (22), and the additivity property of fixed point index that

$$
\begin{aligned}
i\left(A, P \cap\left(T_{r} \backslash \bar{T}_{\rho_{1}}\right), P\right)= & i\left(A, P \cap T_{r}, P\right) \\
& -i\left(A, P \cap T_{\rho_{1}}, P\right)=1-0=1, \\
i\left(A, P \cap\left(T_{\rho_{2}} \backslash \bar{T}_{r}\right), P\right)= & i\left(A, P \cap T_{\rho_{2}}, P\right) \\
& -i\left(A, P \cap T_{r}, P\right)=0-1=-1 .
\end{aligned}
$$

Hence $A$ has at least two fixed points $u_{1}$ and $u_{2}$ in $P \cap\left(T_{r} \backslash \bar{T}_{\rho_{1}}\right)$ and $P \cap\left(T_{\rho_{2}} \backslash \bar{T}_{r}\right)$, respectively. It is obvious that $u_{1}$ and $u_{2}$ are both positive. Moreover, it follows from (14), (21), (23), and the additivity property of fixed point index that

$$
\begin{gathered}
i\left(A,-P \cap\left(T_{r} \backslash \bar{T}_{\rho_{1}}\right),-P\right)=1, \\
i\left(A,-P \cap\left(T_{\rho_{2}} \backslash \bar{T}_{r}\right),-P\right)=-1 .
\end{gathered}
$$

Consequently, $A$ also has at least two fixed points $u_{3}$ and $u_{4}$ in $-P \cap\left(T_{r} \backslash \bar{T}_{\rho_{1}}\right)$ and $-P \cap\left(T_{\rho_{2}} \backslash \bar{T}_{r}\right)$, respectively. Evidently, $u_{3}$ and $u_{4}$ are both negative.

Since $A(P \backslash\{\theta\}) \subset P^{\circ}, A(-P \backslash\{\theta\}) \subset-P^{\circ}$. Let

$$
\begin{gathered}
\Sigma_{1}=\left\{u \in P \cap\left(T_{r} \backslash \bar{T}_{\rho_{1}}\right): A u=u\right\}, \\
\Sigma_{2}=\left\{u \in P \cap\left(T_{\rho_{2}} \backslash \bar{T}_{r}\right): A u=u\right\}, \\
\Sigma_{3}=\left\{u \in-P \cap\left(T_{r} \backslash \bar{T}_{\rho_{1}}\right): A u=u\right\}, \\
\Sigma_{4}=\left\{u \in-P \cap\left(T_{\rho_{2}} \backslash \bar{T}_{r}\right): A u=u\right\} .
\end{gathered}
$$


By Lemma 9 and (24)-(25), we get that there exist open sets $\Omega_{i}(i=1,2,3,4)$ of $E$ such that

$$
\begin{gathered}
\Sigma_{1} \subset \Omega_{1} \subset P \cap\left(T_{r} \backslash \bar{T}_{\rho_{1}}\right), \\
\Sigma_{2} \subset \Omega_{2} \subset P \cap\left(T_{\rho_{2}} \backslash \bar{T}_{r}\right), \\
\Sigma_{3} \subset \Omega_{3} \subset-P \cap\left(T_{r} \backslash \bar{T}_{\rho_{1}}\right), \\
\Sigma_{4} \subset \Omega_{4} \subset-P \cap\left(T_{\rho_{2}} \backslash \bar{T}_{r}\right), \\
\operatorname{deg}\left(I-A, \Omega_{1}, \theta\right)=1, \\
\operatorname{deg}\left(I-A, \Omega_{2}, \theta\right)=-1, \\
\operatorname{deg}\left(I-A, \Omega_{3}, \theta\right)=1, \\
\operatorname{deg}\left(I-A, \Omega_{4}, \theta\right)=-1 .
\end{gathered}
$$

By Lemma 10, we have

$$
\operatorname{deg}\left(I-A, T_{r}, \theta\right)=1 .
$$

According to (32), (28), (30), (18), and the additivity property of Leray-Schauder degree, we obtain

$$
\begin{aligned}
\operatorname{deg}( & \left.I-A, T_{r} \backslash\left(\bar{\Omega}_{1} \cup \bar{\Omega}_{3} \cup \bar{T}_{\rho_{1}}\right), \theta\right) \\
& =1-1-1-1=-2,
\end{aligned}
$$

which yields that $A$ has at least one fixed point $u_{5}$ in $T_{r} \backslash\left(\bar{\Omega}_{1} \cup\right.$ $\left.\bar{\Omega}_{3} \cup \bar{T}_{\rho_{1}}\right)$, and then $u_{5}$ is a sign-changing fixed point. It follows from (19), (29), (31), (32), and the additivity property of LeraySchauder degree that we have

$$
\begin{aligned}
& \operatorname{deg}\left(I-A, T_{\rho_{2}} \backslash\left(\bar{\Omega}_{2} \cup \bar{\Omega}_{4} \cup \bar{T}_{r}\right), \theta\right) \\
& \quad=1+1+1-1=2,
\end{aligned}
$$

which implies that $A$ has at least one fixed point $u_{6}$ in $T_{\rho_{2}} \backslash$ $\left(\bar{\Omega}_{2} \cup \bar{\Omega}_{4} \cup \bar{T}_{r}\right)$, and then $u_{6}$ is also a sign-changing fixed point. The proof is completed.

\section{Example}

The main purpose of this section is to apply our theorem to nonlinear differential equations.

We consider the following boundary value problem for elliptic partial differential equations

$$
\begin{gathered}
L \varphi(x)=f(x, \varphi(x)), \quad x \in \Omega, \\
B \varphi=0, \quad x \in \partial \Omega,
\end{gathered}
$$

where $\Omega$ is a bounded open domain in $\mathbb{R}^{n}, \partial \Omega \in C^{2+\mu}$, and $0<\mu<1 ; f(x, \varphi): \bar{\Omega} \times \mathbb{R}^{1} \rightarrow \mathbb{R}^{1}$ is continuous;

$$
L \varphi=-\sum_{i, j=1}^{n} a_{i j}(x) \frac{\partial^{2} \varphi}{\partial x_{i} \partial x_{j}}+\sum_{i=1}^{n} b_{i}(x) \frac{\partial \varphi}{\partial x_{i}}+c(x) \varphi
$$

is an uniformly elliptic operator; that is, $a_{i j}(x)=a_{j i}(x)$, $b_{i}(x), c(x) \in C^{\mu}(\bar{\Omega}), c(x)>0$ and there exists a constant number $\mu_{0}>0$ such that $\sum_{i, j=1}^{n} a_{i j}(x) \xi_{i} \xi_{j} \geq \mu_{0}|\xi|^{2}$ for all $x \in \bar{\Omega}, \xi=\left(\xi_{1}, \xi_{2}, \ldots, \xi_{n}\right) \in \mathbb{R}^{n}$. Consider

$$
B \varphi=b(x) \varphi+\delta \sum_{i=1}^{n} \beta_{i}(x) \frac{\partial \varphi}{\partial x_{i}}
$$

which is a boundary operator, where $\beta=\left(\beta_{1}, \beta_{2}, \ldots, \beta_{n}\right)$ is a vector field on $\partial \Omega$ of $C^{1+\mu}$ satisfying $\beta \cdot \mathbf{n}>0$ ( $\mathbf{n}$ denotes the outer unit normal vector on $\partial \Omega$ ) and $b(x) \in C^{1+\mu}(\partial \Omega)$, and assume that one of the following cases holds:

(i) $\delta=0$ and $b(x) \equiv 1$;

(ii) $\delta=1$ and $b(x) \equiv 0$;

(iii) $\delta=1$ and $b(x)>0$.

According to the theory of elliptic partial differential equations (see $[20,21]$ ), we know that for each $u \in C(\bar{\Omega})$, the linear boundary value problem

$$
\begin{gathered}
L \varphi(x)=u(x), \quad x \in \Omega, \\
B \varphi=0, \quad x \in \partial \Omega,
\end{gathered}
$$

has a unique solution $\varphi_{u} \in C^{2}(\bar{\Omega})$. Define the operator $K$ by

$$
(K u)(x)=\varphi_{u}(x), \quad x \in \Omega \text {. }
$$

Then $K: C(\bar{\Omega}) \rightarrow C^{2}(\bar{\Omega})$ is a linear completely continuous operator and has an unbounded sequence of eigenvalues:

$$
0<\lambda_{1}<\lambda_{2} \leq \lambda_{3} \leq \cdots, \lambda_{n} \longrightarrow+\infty
$$

and the spectral radius $r(K)=\lambda_{1}^{-1}$.

Let

$$
E=C(\bar{\Omega}), \quad P=\{\varphi \in E \mid \varphi(x) \geq 0, x \in \bar{\Omega}\} .
$$

Then $E$ is an ordered Banach space with the norm $\|\varphi\|=$ $\sup _{x \in \bar{\Omega}}|\varphi(x)|$ and $P$ is a normal solid cone in $E$ and $K(P) \subset P$.

For $\varphi \in E$, define Nemytskii operator by

$$
(F \varphi)(x)=f(x, \varphi(x)), \quad x \in \bar{\Omega} .
$$

Clearly, $F: E \rightarrow E$ is continuous. Let $A=K F$. Then $A$ : $E \rightarrow E$ is completely continuous. $f_{0} K$.

By the proof of Lemma 4.1 in [22], we have that $A_{\theta}^{\prime}=$

Let $e=e(x)$ be the solution of the following boundary value problem

$$
\begin{gathered}
L \varphi(x)=1, \quad x \in \Omega, \\
B \varphi=0, \quad x \in \partial \Omega .
\end{gathered}
$$

In order to obtain multiple sign-changing solutions of (35), we give the following assumptions. 
$\left(\mathrm{E}_{1}\right) f(x, 0) \equiv 0$ and $\forall x \in \bar{\Omega} ; f(x, \varphi) \varphi>0, \forall x \in \bar{\Omega}, \varphi \neq 0$, and $\varphi \in(-\infty,+\infty)$;

$\left(\mathrm{E}_{2}\right) \lim _{\varphi \rightarrow 0}(f(x, \varphi) / \varphi)=f_{0}$ uniformly for $x \in \bar{\Omega}$, and $\lambda_{n}<f_{0}<\lambda_{n+1}$, where $n$ is an even number;

$\left(\mathrm{E}_{3}\right) \lim _{\varphi \rightarrow \infty}(f(x, \varphi) / \varphi)=f_{\infty}$ uniformly for $x \in \bar{\Omega}$, and $\lambda_{n_{0}}<f_{\infty}<\lambda_{n_{0}+1}$, where $n_{0}$ is an even number;

$\left(\mathrm{E}_{4}\right)$ there exists a constant number $r<1$ such that $\lim _{\varphi \rightarrow 0}(f(x, \varphi) / \varphi)=r(1 /\|e\|)$ uniformly for $x \in \bar{\Omega}$.

Theorem 12. Suppose that $\left(E_{1}\right)-\left(E_{4}\right)$ are satisfied. Then the problem (35) has at least two sign-changing solutions. Moreover, problem (35) has at least two positive solutions and two negative solutions.

Proof. From condition $\left(\mathrm{E}_{1}\right)$, we know that

$$
\begin{aligned}
& f(x, \varphi)>0, \quad \forall \varphi>0 ; \\
& f(x, \varphi)<0, \quad \forall \varphi<0 .
\end{aligned}
$$

Copy the proof proceed of Theorem 3.4 in [23], we have that $A\left(\left(E_{e_{0}} \cap P\right) \backslash\{\theta\}\right) \subset \operatorname{int}\left(E_{e_{0}} \cap P\right)$, and $A\left(-\left(E_{e_{0}} \cap P\right) \backslash\{\theta\}\right) \subset$ $\operatorname{int}\left(-\left(E_{e_{0}} \cap P\right)\right)$, where

$$
\begin{gathered}
E_{e_{0}}=\{\varphi \in E \text { : there exists } \mu>0 \\
\text { such that } \left.-\mu e_{1}(x) \leq \varphi \leq \mu e_{1}(x)\right\}, \\
\operatorname{int~}\left(E_{e_{0}} \cap P\right)=\left\{\varphi \in\left(E_{e_{0}} \cap P\right) \text { : there exist } \alpha>0\right. \\
\text { and } \beta>0 \text { such that } \alpha e_{1}(x) \\
\left.\leq \varphi(x) \leq \beta e_{1}(x)\right\},
\end{gathered}
$$

where $e_{1}$ is the first normalized eigenfunction of $K$ corresponding to its first eigenvalue $\lambda_{1}$.

It follows from $\left(\mathrm{E}_{2}\right)$ and $\left(\mathrm{E}_{3}\right)$ that conditions $\left(\mathrm{H}_{1}\right)$ and $\left(\mathrm{H}_{2}\right)$ of Theorem 11 hold.

In the following, we prove that $\left(\mathrm{H}_{3}\right)$ of Theorem 11 is satisfied. It follows from $\left(\mathrm{E}_{4}\right)$ that there exists $\delta>0$ such that

$$
\frac{f(x, \varphi)}{\varphi}<\frac{1+r}{2\|e\|}, \quad \forall 0<|\varphi|<\delta, x \in \bar{\Omega} .
$$

That is,

$$
|f(x, \varphi)|<\frac{1+r}{2\|e\|}|\varphi|, \quad x \in \bar{\Omega} .
$$

Thus

$$
\|A \varphi\| \leq \frac{1+r}{2\|e\|}\|e\|\|\varphi\|
$$

Therefore,

$$
\lim _{\|\varphi\| \rightarrow 0} \frac{\|A \varphi\|}{\|\varphi\|} \leq \frac{1+r}{2}<1 .
$$

The proof is completed.

Remark 13. It follows from conditions $\left(\mathrm{E}_{2}\right)$ and $\left(\mathrm{E}_{4}\right)$ that $f_{0}\|e\|<1$. We should point out that the initial ideas of condition $\left(\mathrm{E}_{4}\right)$ and the general one $\left(\mathrm{H}_{3}\right)$ are motivatedby [24].

\section{Conflict of Interests}

The author declares that there is no conflict of interests regarding the publication of this paper.

\section{Acknowledgment}

The author was supported financially by the National Natural Science Foundation of China, Tianyuan Foundation (11226119), the Scientific and Technological Innovation Programs of Higher Education Institutions in Shanxi, the Youth Science Foundation of Shanxi Province (2013021002-1), and Shandong Provincial Natural Science Foundation, China (ZR2012AQ024).

\section{References}

[1] E. N. Dancer and Y. H. Du, "Existence of changing sign solutions for some semilinear problems with jumping nonlinearities at zero," Proceedings of the Royal Society of Edinburgh, vol. 124, no. 6, pp. 1165-1176, 1994.

[2] D. Motreanu and M. Tanaka, "Sign-changing and constant-sign solutions for $p$-Laplacian problems with jumping nonlinearities," Journal of Differential Equations, vol. 249, no. 12, pp. 33523376,2010

[3] Z. Zhang and S. Li, "On sign-changing and multiple solutions of the $p$-Laplacian," Journal of Functional Analysis, vol. 197, no. 2, pp. 447-468, 2003.

[4] W. Zou, Sign-Changing Critical Point Theory, Springer, New York, NY, USA, 2008.

[5] X. Cheng and C. Zhong, "Existence of three nontrivial solutions for an elliptic system," Journal of Mathematical Analysis and Applications, vol. 327, no. 2, pp. 1420-1430, 2007.

[6] K. Zhang, The multiple solutions and sign-changing solutions for nonlinear operator equations and applications [Ph.D. thesis], Shandong University, Jinan, China, 2002 (Chinese).

[7] X. Xu, "Multiple sign-changing solutions for some m-point boundary-value problems," Electronic Journal of Differential Equations, vol. 89, pp. 1-14, 2004.

[8] R. Yang, Existence of positive solutions, sign-changing solutions for nonlinear differential equations [M.S. thesis], Shandong University, Jinan, China, 2006 (Chinese).

[9] C. Pang, W. Dong, and Z. Wei, "Multiple solutions for fourthorder boundary value problem," Journal of Mathematical Analysis and Applications, vol. 314, no. 2, pp. 464-476, 2006.

[10] Z. Wei and C. Pang, "Multiple sign-changing solutions for fourth order $m$-point boundary value problems," Nonlinear Analysis: Theory, Methods \& Applications, vol. 66, no. 4, pp. 839855, 2007.

[11] Y. Li and F. Li, "Sign-changing solutions to second-order integral boundary value problems," Nonlinear Analysis: Theory, Methods \& Applications, vol. 69, no. 4, pp. 1179-1187, 2008.

[12] T. He, W. Yang, and F. Yang, "Sign-changing solutions for discrete second-order three-point boundary value problems," Discrete Dynamics in Nature and Society, Article ID 705387, 14 pages, 2010.

[13] J. Yang, "Sign-changing solutions to discrete fourth-order Neumann boundary value problems," Advances in Difference Equations, vol. 2013, article 10, 2013.

[14] K. Deimling, Nonlinear Functional Analysis, Springer, Berlin, Germany, 1985. 
[15] D. Guo, Partial Order Methods in Nonlinear Analysis, Shandong Science and Technology Press, Jinan, China, 2000 (Chinese).

[16] D. Guo, Nonlinear Functional Analysis, Shandong Science and Technology Press, Jinan, China, 2nd edition, 2001 (Chinese).

[17] F. Li, Solutions of nonlinear operator equations and applications [Ph.D. thesis], Shandong University, Jinan, China, 1996 (Chinese).

[18] J. Sun, Nonlinear Functional Analysis and Applications, Science Press, Beijing, China, 2008 (Chinese).

[19] H. Amann, "Fixed points of asymptotically linear maps in ordered Banach spaces," Journal of Functional Analysis, vol. 14, pp. 162-171, 1973.

[20] H. Amann, "On the number of solutions of nonlinear equations in ordered Banach spaces," Journal of Functional Analysis, vol. 11, pp. 346-384, 1972.

[21] H. Amann, "Fixed point equations and nonlinear eigenvalue problems in ordered Banach spaces," SIAM Review, vol. 18, no. 4, pp. 620-709, 1976.

[22] X. Liu and J. Sun, "Computation of topological degree of unilaterally asymptotically linear operators and its applications," Nonlinear Analysis: Theory, Methods \& Applications, vol. 71, no. 1-2, pp. 96-106, 2009.

[23] J. X. Sun and X. Y. Liu, "Computation of the fixed point index for non-cone mappings and its applications," Acta Mathematica Sinica, vol. 53, no. 3, pp. 417-428, 2010 (Chinese).

[24] J. X. Sun and K. M. Zhang, "Existence of multiple fixed points for nonlinear operators and applications," Acta Mathematica Sinica, vol. 24, no. 7, pp. 1079-1088, 2008. 


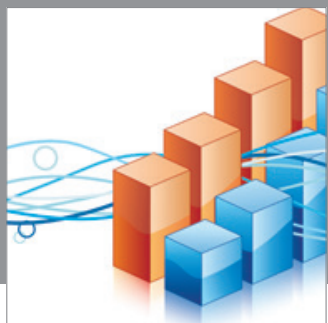

Advances in

Operations Research

mansans

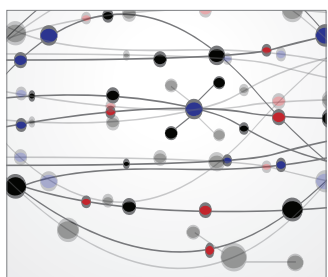

The Scientific World Journal
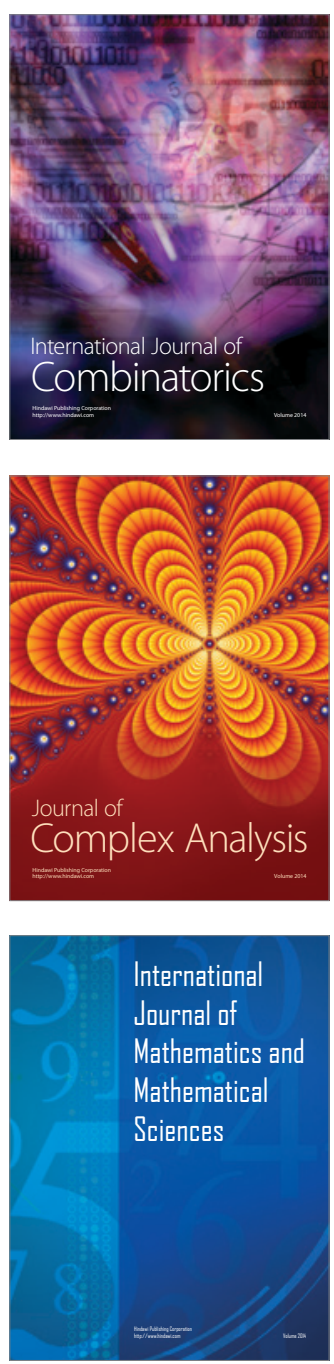
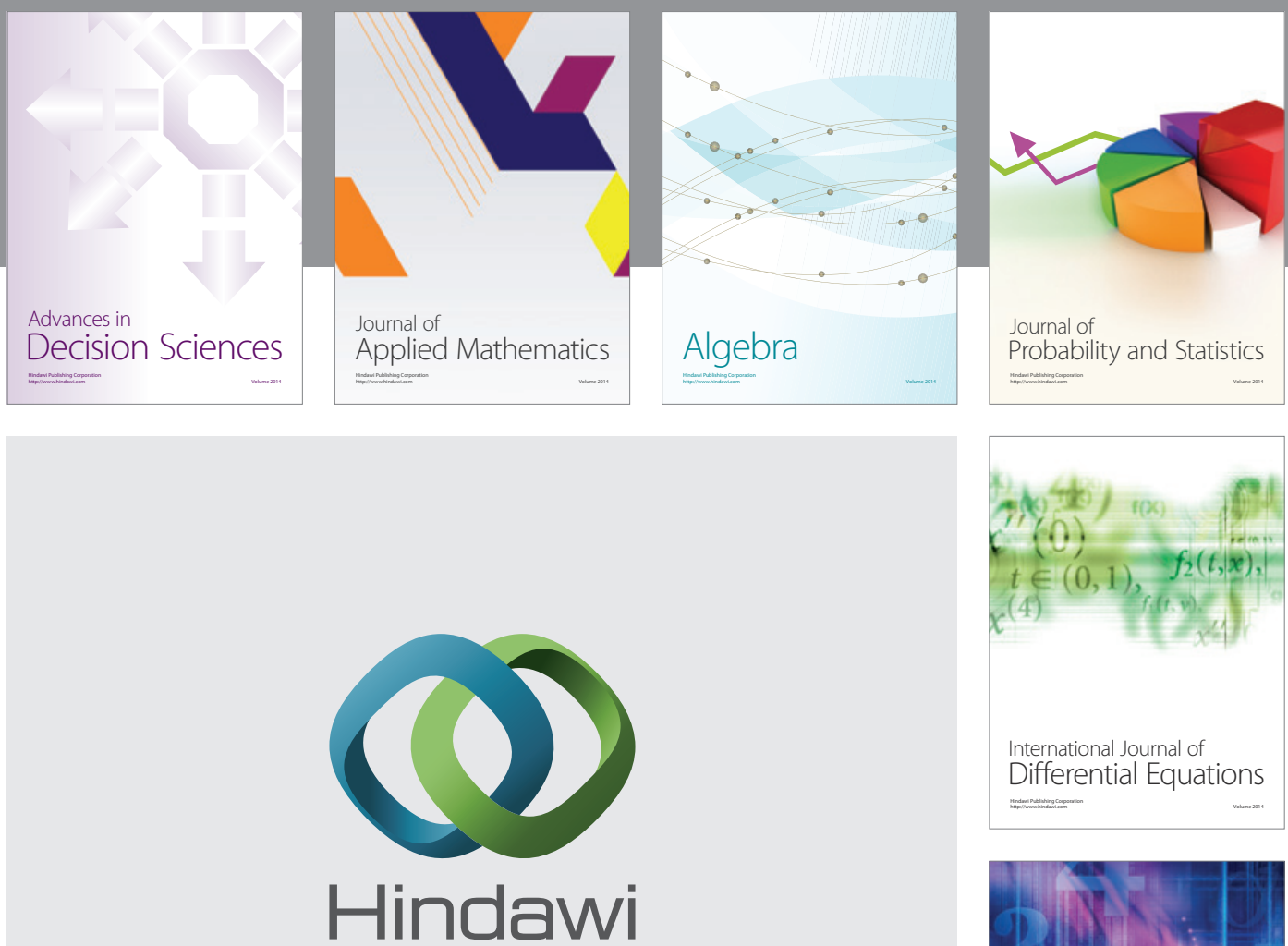

Submit your manuscripts at http://www.hindawi.com
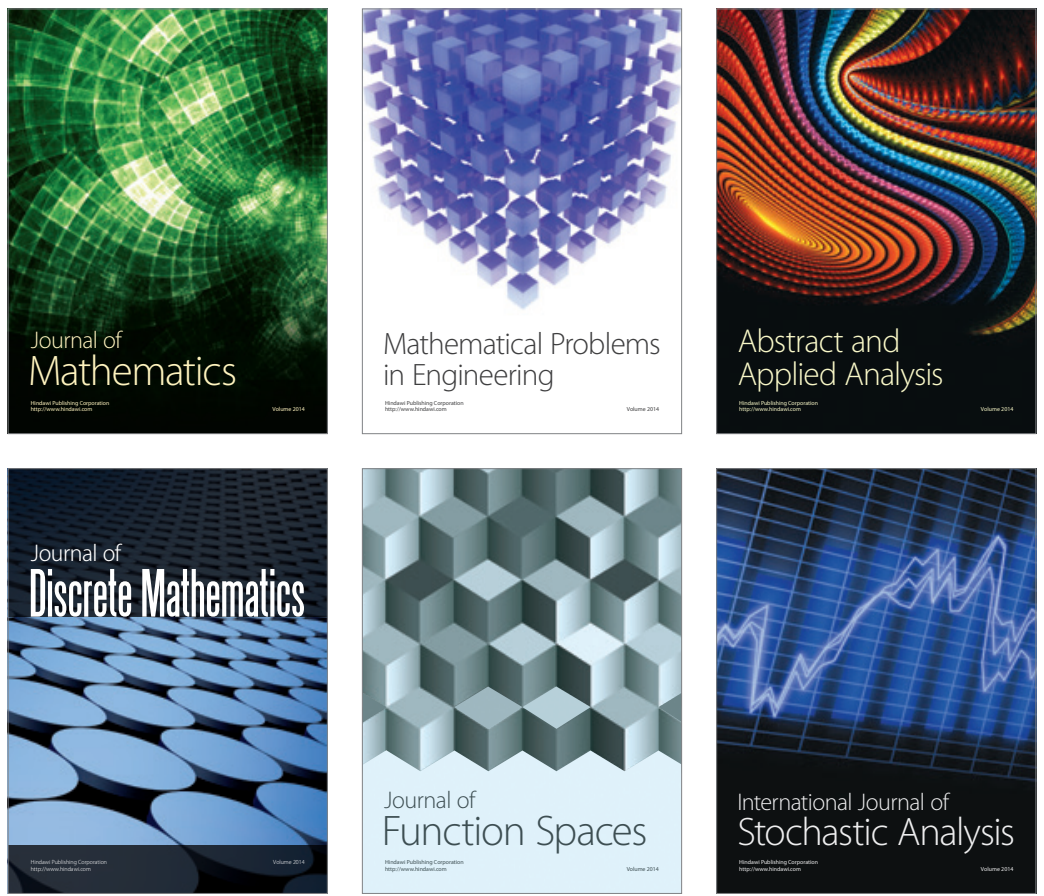

Journal of

Function Spaces

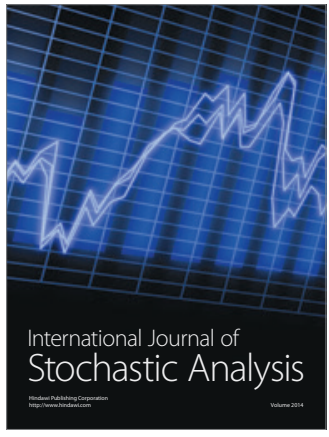

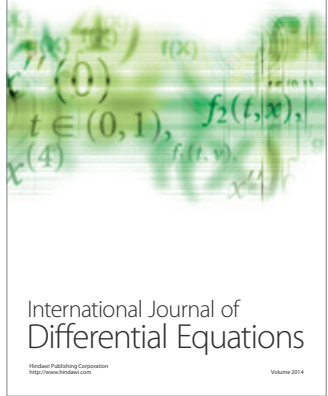
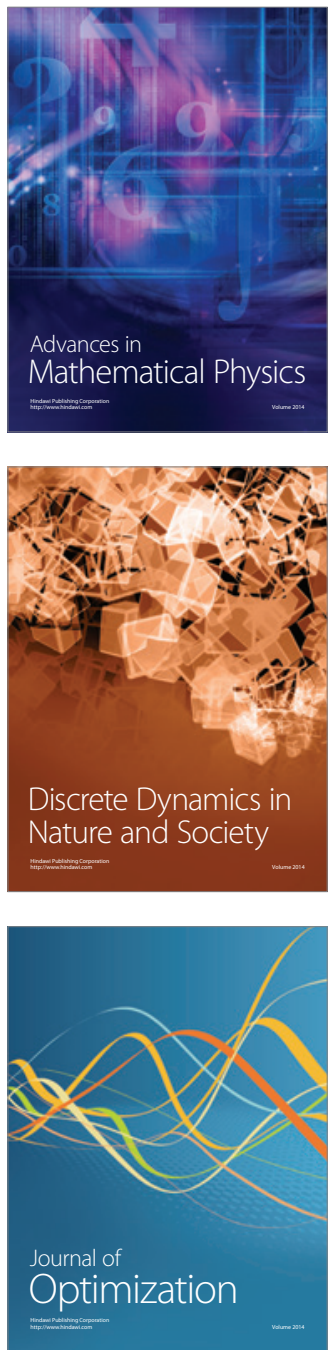